corresponds to an accelerating electric field of $2.8 \mathrm{GV} \mathrm{m}^{-1}$, which is the largest coherent man-made accelerating field yet produced, and 30 times larger than the limit imposed by radiofrequency breakdown in conventional accelerating structures. If the beat wave could be $10 \mathrm{~cm}$ long rather than $1 \mathrm{~cm}$ as in the experiment, electrons would reach an energy of $300 \mathrm{MeV}$.

Even if such 'bench-top' machines prove unable to compete with the present high-energy colliders, they could proliferate in research laboratories just as computers have with the advent of PCs and mini-workstations. Apart from their compactness, these plasma accelerators have the interesting feature that they produce a series of very short electron bunches separated by a plasma wave period - here, 1 picosecond $\left(10^{-12} \mathrm{~s}\right)$. These periodic electron pulses could be used to make tiny stroboscopic X-ray or light sources, opening up the prospect of making slowmotion movies of chemical reactions or dynamical biological processes that have never been observed before. In addition, the tiny bursts of X-rays might permit better imaging in biology and medicine using only a fraction of the usual radiation.

In obtaining their results, Joshi and colleagues have overcome some tough practical problems. Their plasma is uniform in density to within 1 per cent, a remarkable value achieved by tunnel ionization of a static fill of hydrogen gas. Essentially the kinetic energy of the electrons increases because of the electric field of the laser - they oscillate in the laser's field. When the laser intensity reaches a critical threshold value in the gas, electrons bound to the nucleus tunnel through the Coulomb barrier, becoming free. The ionization time is of the order of the laser period $\left(10^{-15} \mathrm{~s}^{-1}\right)$ which is extremely small.

The tendency for strong plasma turbulence to develop, destroying the coherency of the accelerating fields, was overcome by lowering the plasma density, which effectively increased the growth time of plasma instabilities. With the laser pulse and electrons moving close to the speed of light through the plasma, turbulence effects do not have time to establish before the pulse and electrons have passed on.

The work on plasma-based accelerators represents but one area that is being explored by researchers in the advanced accelerator field. Other schemes being investigated at present for high-gradient acceleration are the inverse Cerenkov effect and the inverse free-electron laser effect. Still other researchers, realizing that the next collider will almost certainly be a linear electron-positron collider, are proposing a novel way of building such a device known as a two-beam accelerator.

\title{
Laser acceleration of particles
}

IN laser beat-wave experiments, as car. ried out by Joshi and colleagues, two laser frequencies are incident on a hydrogen plasma producing regions of constructive and destructive interference. The result is a series of light pulses

velocities are close to the speed of light $c$ in a vacuum: $v_{\mathrm{p}}=v_{\mathrm{g}}=c(1-$ $\left.\omega_{\text {pe }}{ }^{2} / \omega^{2}\right)^{1 / 2}$. Because $\omega$ is close to $\omega^{\prime}$ and much larger than $\omega_{\text {pe, }}$ the Lorentz factor $\gamma_{\mathrm{p}}$ associated with the beat waves is $\left[1-v_{\mathrm{p}}^{2} / c^{2}\right]^{-1 / 2}=\omega / \omega_{\mathrm{pe}} \gg 1$.
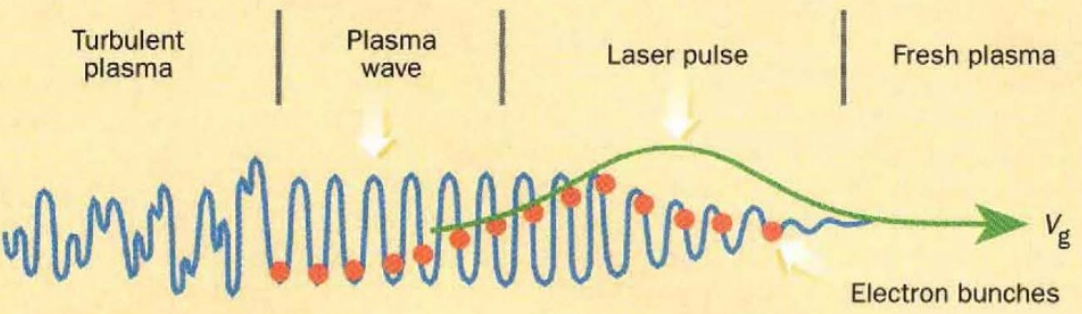

moving through the plasma at the group velocity of light.

The plasma electrons feel periodic electromagnetic pressure forces at a frequency corresponding to the difference of the two laser frequencies. If this difference frequency equals the natural oscillation frequency of the electron plasma, $\omega_{\mathrm{pe}}\left(=5.64 \times 10^{4} \mathrm{n}_{\mathrm{e}}{ }^{1 / 2} \mathrm{rad} \mathrm{s}^{-1}\right.$, where $n_{\mathrm{e}}$ is the electron plasma density in units of electrons per cubic centimetre) the plasma responds resonantly to these forces and large-amplitude plasma osciliations build up, as shown schematically above.

The laser wakefield scheme is rather different. There, a single laser pulse less than half the length of an electron plasma wave leaves a wake of relativistic electron plasma oscillations.

In both schemes, the electron plasma waves have a phase velocity, $v_{p}$, which is equal to the group velocity of light $v_{\mathrm{g}}$ in the plasma. For laser frequencies $\omega$ and $\omega^{\prime}$ that are much larger than the plasma frequency, which is the case in Joshi and colleagues' experiment, these

And there are many groups developing an entirely new type of electron lens using focusing by a plasma to increase the luminosity of future linear colliders ${ }^{4}$.

This plays on the fact that relativistic electron beams can be focused by a plasma if the collisionless skin depth $c / \omega_{\mathrm{pe}}$ is larger than the beam radius. Generally, when a relativistic electron beam enters a plasma, the plasma electrons move to neutralize the charge in the beam on a $1 / \omega_{\mathrm{pe}}$ timescale. However, if the collisionless skin depth is larger than the beam radius, the axial return current flows in the plasma on the outside of the electron beam and the beam current is not fully neutralized, leading to the generation of an azimuthal magnetic field. Consequently this self-generated magnetic field pinches or focuses the beam in the radial direction. This type of lens exceeds con-
The longitudinal electric field amplitude of these relativistic plasma waves can be extremely large, with a theoretifor plasma densities of the order of $10^{18}$ $\mathrm{cm}^{-3}$, the field strength can be $10^{9} \mathrm{~V}$ $\mathrm{cm}^{-1}$, equivalent to fields in atoms.

In practice this maximum wave electric field value, set by wave-breaking, is never achieved because the relativistic mass increase of the oscillating electrons changes the plasma frequency and resonance is lost. So the maximum energy to which an electron can be accelerated is $\boldsymbol{W}=2 \varepsilon \gamma_{\mathrm{p}}^{2} m_{\mathrm{e}} c^{2}$ where $\varepsilon$ is the ratio of the plasma wave amplitude to its theoretical maximum. As an example, if we take a plasma density of $10^{19} \mathrm{~cm}^{-3}$ and $\varepsilon=0.3,30$ per cent of the maximum value, results in an electric field of about $10^{9} \mathrm{~V} \mathrm{~cm}^{-1}$ - capable of producing a $\mathrm{GeV}$ electron in a distance of $1 \mathrm{~cm}$. Joshi and colleagues no doubt have their sights set on this next step, although higher-frequency lasers than $\mathrm{CO}_{2}$ will have to be used in these denser plasmas.

R. B.

ventional lenses by several orders of magnitude in focusing gradient.

For the beat-wave scheme, the next milestone to be achieved is the $\mathrm{GeV}$ energy level, and already Joshi and his team are planning this next step. It is indeed gratifying to see that some of the ideas on alternative acceleration schemes proposed more than a decade ago are coming to fruition.

Robert Bingham is at the Rutherford Appleton Laboratory, Chilton, Didcot, Oxfordshire OX11 OQX, UK.

1. Everett, M., Lal, A., Gordon, D., Clayton, C. E., Marsh, K. A. \& Joshi, C. Nature 368, 527-529 (1994).

2. Tajima, T. \& Dawson, J. M. Phys. Rev. Lett. 43, 267-270 (1979)

3. Katsouleas, T. et al. Proc. AlP Conf. No. 130 Laser Accelerations of Particles (eds Joshi, C. \& Katsouleas, T.) 663-698 (American Institute of Physics, 1985).

4. Chen, P. Particle Accel. 20, 171 (1987). cal maximum given by $E=n_{e}^{1 / 2} \mathrm{~V} \mathrm{~cm}^{-1}$; 